\title{
Targeted deep sequencing in polycythemia vera and essential thrombocythemia
}

Ayalew Tefferi, ${ }^{1}$ Terra L. Lasho, ${ }^{1}$ Paola Guglielmelli, ${ }^{2}$ Christy M. Finke, ${ }^{1}$ Giada Rotunno,${ }^{2}$ Yoseph Elala, ${ }^{1}$ Annalisa Pacilli, ${ }^{2}$ Curtis A. Hanson, ${ }^{3}$ Alessandro Pancrazzi, ${ }^{2}$ Rhett P. Ketterling, ${ }^{4}$ Carmela Mannarelli, ${ }^{2}$ Daniela Barraco, ${ }^{1}$ Tiziana Fanelli, ${ }^{2}$ Animesh Pardanani, ${ }^{1}$ Naseema Gangat, ${ }^{1}$ and Alessandro M. Vannucchi ${ }^{2}$

${ }^{1}$ Division of Hematology, Department of Internal Medicine, Mayo Clinic, Rochester, MN; ${ }^{2}$ Centro Ricerca e Innovazione Malattie Mieloproliferative, Azienda Ospedaliera Universitaria Careggi, and Department of Experimental and Clinical Medicine, University of Florence, Florence, Italy; and ${ }^{3}$ Division of Hematopathology and ${ }^{4}$ Division of Cytogenetics, Department of Laboratory Medicine, Mayo Clinic, Rochester, MN

\section{Key Points}

- More than half of patients with PV or ET harbor DNA mutations/ variants other than JAK2/CALR/MPL.

- The presence of some of these mutations adversely affects overall, leukemia-free, or myelofibrosis-free survival.
Polycythemia vera (PV) is characterized by JAK2 and essential thrombocythemia (ET) by JAK2, calreticulin (CALR), and myeloproliferative leukemia virus oncogene (MPL) mutations; we describe the occurrence and prognostic relevance of DNA sequence variants/mutations other than JAK2/CALR/MPL. A myeloid neoplasm-relevant 27-gene panel was used for nextgeneration sequencing of bone marrow or whole blood DNA and conventional tools were used for analysis. "Adverse variants/mutations" were identified by age-adjusted multivariable analysis of impact on overall, leukemia-free, or myelofibrosis-free survival. Fifty-three percent of 133 Mayo Clinic patients with PV and 53\% of 183 with ET harbored 1 or more sequence variants/mutations other than JAK2/CALR/MPL; the most frequent were TET2 and ASXL1. "Adverse variants/mutations" in PV included ASXL1, SRSF2, and IDH2 and in ET SH2B3, SF3B1, U2AF1, TP53, IDH2, and EZH2; combined prevalence was 15\% and 15\%, respectively. Adverse variants/mutations were associated with inferior survival in both PV (median, 7.7 vs 16.9 years) and ET (median, 9 vs 22 years) and the effect was independent of conventional prognostic models with respective hazard ratio (95\% confidence interval) of 2.8 (1.5-5.1) and 2.6 (1.4-4.8); these observations were validated in 215 Italian patients with PV and 174 with ET. In both Mayo Clinic and Italian cohorts, leukemic or fibrotic progression was also predicted by adverse variants/mutations. Number of mutations did not provide additional prognostic information. We conclude that targeted deep sequencing in PV and ET allows for genetic risk stratification that is independent of clinically derived prognostic models.

\section{Introduction}

Despite seminal descriptions of novel ${ }^{1-6}$ and other less specific ${ }^{7,8}$ mutations in the last 10 years, the molecular pathogenesis of myeloproliferative neoplasms (MPNs) remains incompletely understood; however, there has been increasing use of these mutations in diagnostics and disease prognostication. ${ }^{9}$ Mutations in MPNs are operationally classified into those that involve JAK2, calreticulin (CALR), and myeloproliferative leukemia virus oncogene $(M P L)$ and those that involve other genes, such as additional sex combs like transcriptional regulator $1(A S X L 1) .{ }^{9}$ The former are believed to be "driver" mutations and are now formally integrated into the World Health Organization (WHO) diagnostic criteria for polycythemia vera (PV; 98\% JAK2 mutational frequency), essential thrombocythemia (ET; 60\% JAK2, $22 \% C A L R$, and $3 \% M P L$ mutational frequency) and primary myelofibrosis (PMF). ${ }^{10}$ In terms of disease
Submitted 8 August 2016; accepted 6 September 2016. DOI 10.1182/ bloodadvances. 2016000216.
The full-text version of this article contains a data supplement. (c) 2016 by The American Society of Hematology 
prognostication, type 1/type 1-like CALR mutations have been associated with superior survival in $\mathrm{PMF}^{11,12}$ and JAK2 mutations with thrombosis in ET. ${ }^{13}$

The prognostic contribution of mutations other than $J A K 2, C A L R$, or $M P L$ has been demonstrated in PMF, where $A S X L 1$, serine/argininerich splicing factor 2 (SRSF2), isocitrate dehydrogenase 1 or 2 (IDH1/2), and enhancer of zeste 2 polycomb repressive complex 2 subunit $(E Z H 2)$ were identified as being unfavorable to survival. ${ }^{7}$ More recent studies have suggested the prognostic relevance of not only the specific type but also the number of mutations., 8,14 These observations are noteworthy considering the increasing application of next-generation sequencing (NGS) technology in clinical practice. In the current study, we used custom-designed targeted sequencing of 27 myeloid neoplasm-relevant genes that are also often included in most commercially available NGS gene panels used in myeloid cancer. Our main objective was to determine the prognostic and phenotypic relevance of sequence variants/mutations, other than $J A K 2, C A L R$ and $M P L$, in PV and ET.

\section{Methods}

The current study was approved by the institutional review boards of the Mayo Clinic and the University of Florence, Florence, Italy. Diagnoses and treatment approaches were in accordance with what was considered standard of care at the time of initial diagnosis. ${ }^{15}$ Targeted capture assays for Mayo Clinic patients (the main study cohort) were carried out on bone marrow or whole-blood DNA for the following genes: TET2, DNMT3A, IDH1, IDH2, ASXL1, EZH2, SUZ12, SRSF2, SF3B1, ZRSR2, U2AF1, PTPN11, TP53, SH2B3, RUNX1, CBL, NRAS, JAK2, CSF3R, FLT3, KIT, CALR, MPL, NPM1, CEBPA, IKZF1, and SETBP1. Additional details regarding NGS analysis are outlined in the supplemental Methods, which also includes the methods used in the validation cohort from the University of Florence. Prognostic evaluation of sequence variants/mutations considered both the "number" of sequence variants/mutations and the specific genes affected. "Adverse sequence variants/mutations" were identified by age-adjusted multivariable analysis of their impact on overall survival or multivariable analysis of impact on leukemia-free or myelofibrosis-free survival.

All statistical analyses considered clinical and laboratory parameters obtained at time of diagnosis. Differences in the distribution of continuous variables between categories were analyzed by either the Mann-Whitney or the Kruskal-Wallis test. Patient groups with nominal variables were compared by $\chi^{2}$ test. Survival analysis was considered from the date of diagnosis to date of death or last contact; because of the considerable effect of age on survival, univariate analysis for overall survival was adjusted for age. Leukemiafree and myelofibrosis-free survival calculations considered the transformation events as the uncensored variable. Survival curves were prepared by the Kaplan-Meier method and compared by the log-rank test. The Cox proportional hazard regression model was used for multivariable analysis. $P$ values $<.05$ were considered significant. All analyses were conducted using the Stat View (SAS Institute, Cary, NC).

\section{Results}

A total of 316 Mayo Clinic patients with PV $(n=133)$ or ET $(n=183)$ were evaluated; Tables 1 and 2 outline the presenting clinical and laboratory details. "Driver" mutation distribution was 98\% JAK2 for PV and 52\% JAK2, 26\% CALR, and 4\% MPL for ET. According to conventional prognostic models, ${ }^{16,17}$ survival risk distribution at time of diagnosis was 45\% high, 26\% intermediate, and 29\% low for PV, and $21 \%$ high, $39 \%$ intermediate, and $40 \%$ low for ET. Median follow-up was 10.4 years for PV and 10.1 years for ET. The respective numbers of documented deaths, leukemic transformations, and fibrotic progressions were, for PV, 62 (47\%), 7 (5.3\%), and 14 (11\%) and for ET 61 (33\%), 6 (3.3\%), and 27 (15\%).

\section{Prevalence and cosegregation of sequence variants/mutations}

Sequence variants/mutations other than JAK2, CALR, or MPL were seen in 70 patients with PV (52.6\%) and 96 with ET (52.5\%) (Figure 1); supplemental Table 1 provides additional information on specific variants/mutations. The respective percentages of patients with 1,2 , or $\geq 3$ sequence variants/mutations were $30 \%, 20 \%$, and $3 \%$ for PV and $41 \%, 8 \%$, and $4 \%$ for ET. The most frequent sequence variants/mutations in both disorders were TET2 and ASXL1 (Figure 1). There were no significant associations between $J A K 2 / C A L R / M P L$ mutational status and number or type of other sequence variants/mutations. Among the most frequent sequence variants/mutations, $A S X L 1$ in ET cosegregated with EZH2 $(P<.001)$, $I D H 2(P=.004)$, and $R U N X 1(P=.002)$ and in PV with IDH2 $(P=.003)$ and KIT $(P=.02)$; TET2 in PV cosegregated with SH2B3 $(P=.01)$.

\section{Phenotypic correlations of sequence variants/mutations}

Phenotypic correlations were examined for sequence variants/mutations with a recurrence rate of at least $5 \%$ (Figure 1). Parameters examined for possible association included age, sex, risk category, ${ }^{16,17}$ karyotype, thrombosis history, leukocyte count, hemoglobin level, platelet count, palpable splenomegaly, pruritus, and microvascular symptoms. The most noteworthy association was between TET2 variants/mutations and thrombosis in ET $(P=.01)$, which was independent of both age and $J A K 2 / C A L R / M P L$ mutational status; relative risk on age-adjusted multivariable analysis was 3.4 (95\% confidence interval [Cl], 1.4-8.4), compared with $4.8(95 \% \mathrm{Cl}, 1.6-14.2)$ for JAK2 vs CALR. However, such association was not evident in either $\mathrm{PV}$ or an external cohort of 174 Italian patients with ET seen at the University of Florence $(P=.58)$. In ET, TET2 and SF3B1 variants/mutations were associated with older age $(P=.04$ and 0.04 , respectively), SF3B1 with higher platelet count $(P=.02)$, and $A S X L 1$ with palpable splenomegaly $(P=.009)$. In $\mathrm{PV}$, the only association noted was between $\mathrm{SH} 2 B 3$ variants/mutations and palpable splenomegaly $(P=.045)$.

\section{Prognostic relevance of sequence variants/mutations in PV}

Median survival of the 133 Mayo Clinic patients with PV was 14.2 years. Age-adjusted univariate analysis identified SRSF2, ASXL1, and IDH2 variants/mutations as being significantly associated with inferior survival; ASXL1 (hazard ratio [HR], 2.2; 95\% Cl, 1.1-4.3) and SRSF2 $(\mathrm{HR}, 6.1 ; 95 \% \mathrm{Cl}, 2.0-19.2)$ remained significant on multivariable analysis. For leukemia-free and myelofibrosis-free survival, univariate analysis showed significant associations with SRSF2, IDH2, and RUNX1 for the former and with SRSF2 and RUNX1 for the latter; on multivariable analysis, SRSF2 (HR, 74.5, 95\% Cl, 4.4-1261.7) and IDH2 (HR, 55.5; 95\% Cl, 3.5-887.4) remained significant for leukemia-free survival and SRSF2 (HR, 27.2; 95\% Cl, 2.7-274.3) for myelofibrosis-free survival. 


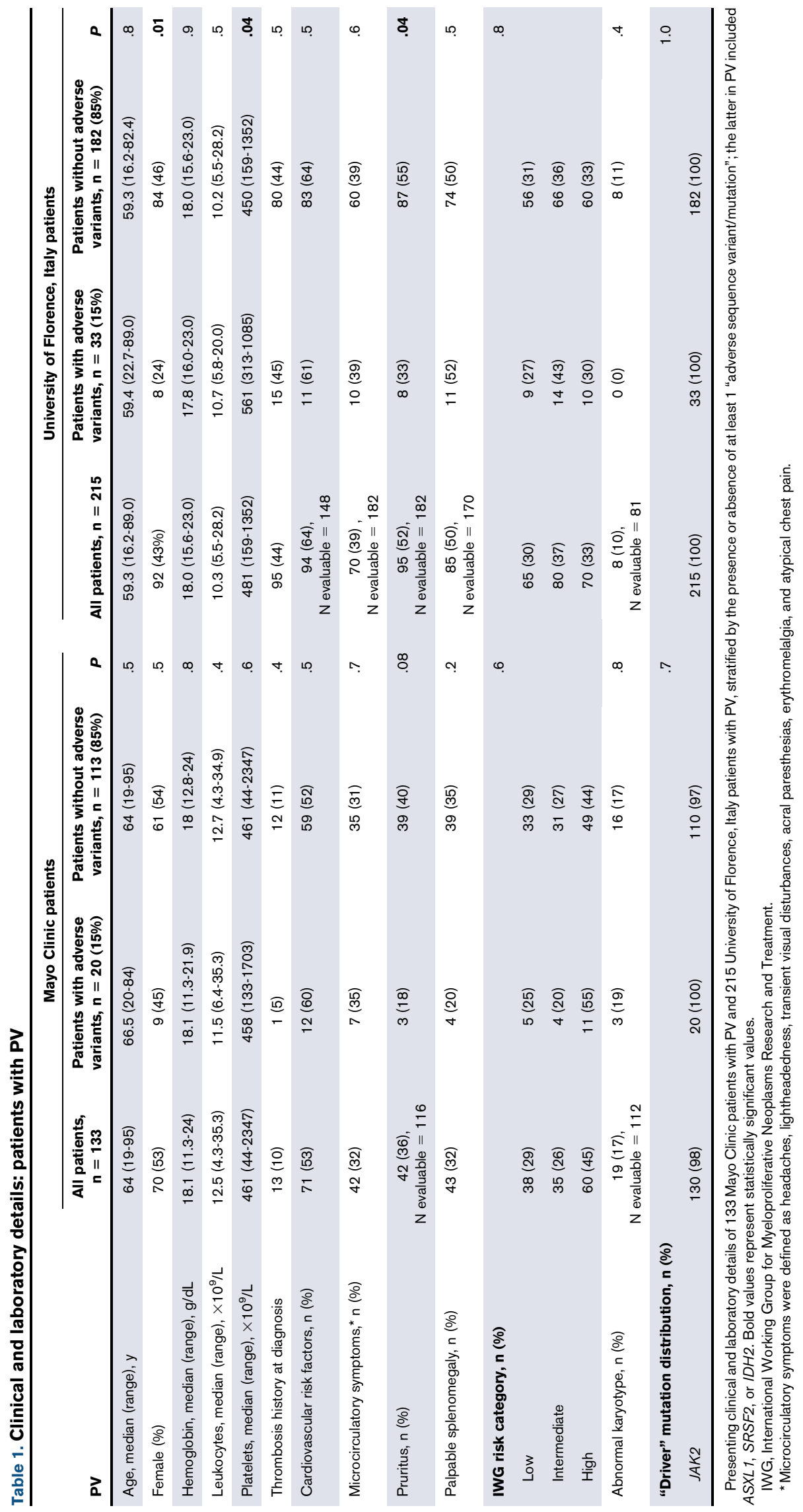




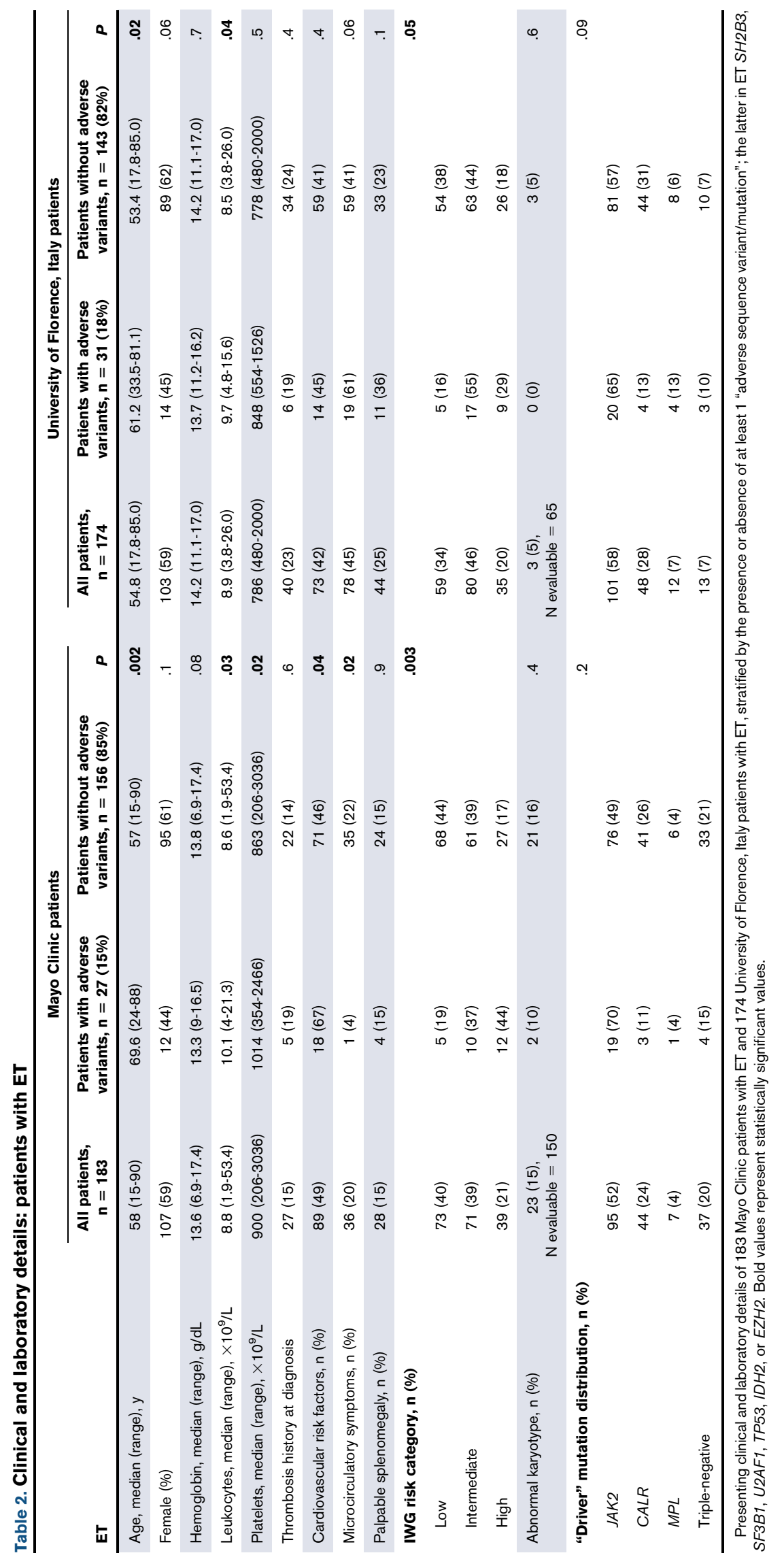




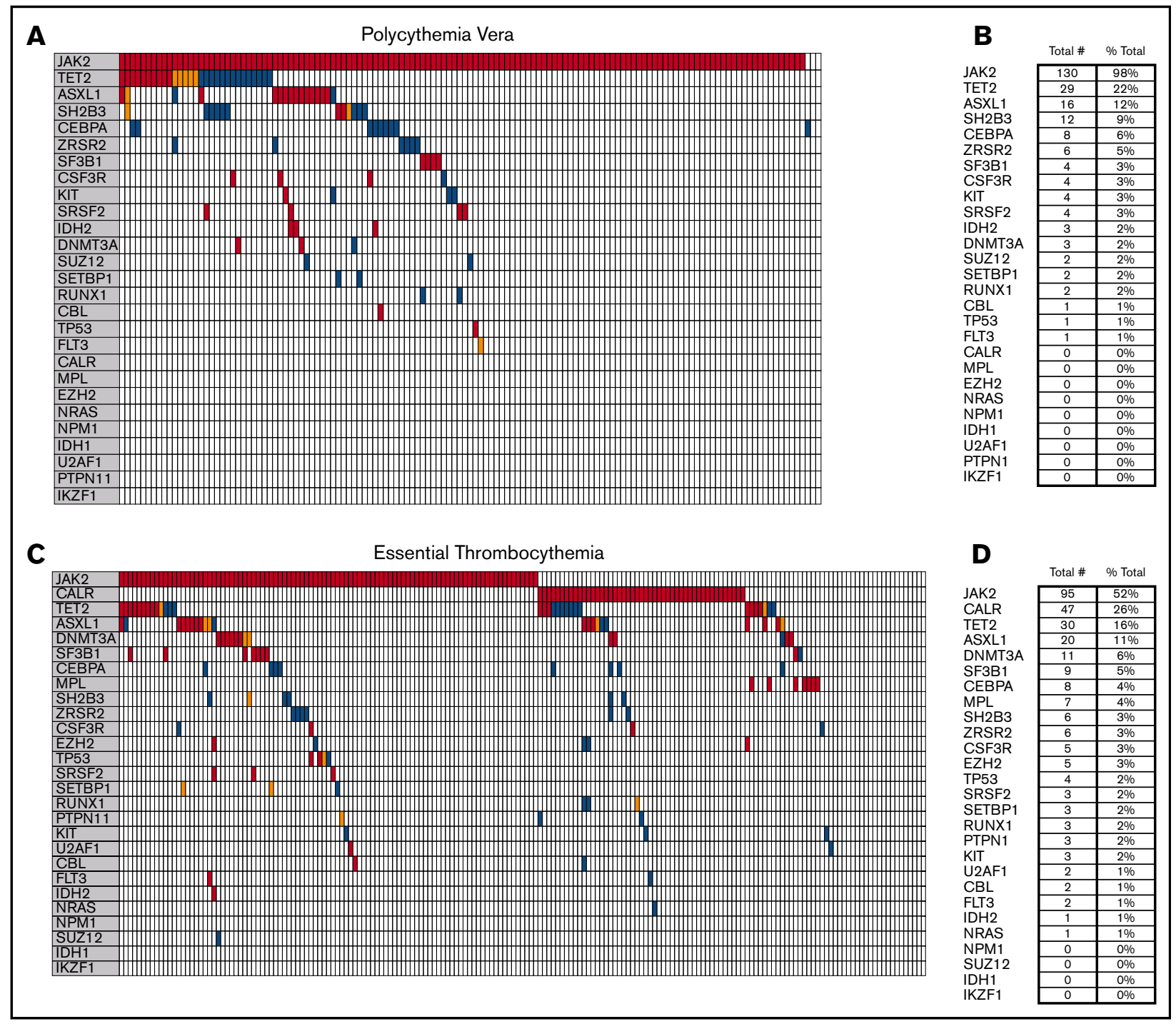

Figure 1. Twenty-seven-gene panel DNA sequence variants in Mayo Clinic patients with $\mathbf{P V}(\mathbf{n}=\mathbf{1 3 3})$ and ET (n $=\mathbf{1 8 3})$. (A,C) Individual variant/mutational cosegregation plot for both PV and ET. Each column represents 1 of the sequenced subjects. Variant/mutations are depicted by representative colored bars. Red, Variants

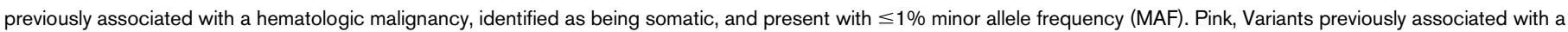

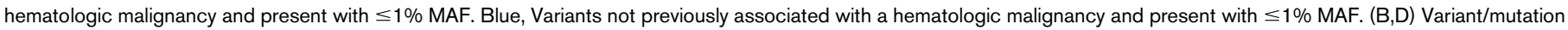
totals in PV and ET ranked by gene and corresponding overall frequency percentage.

Based on their above-outlined independent impact on overall, leukemiafree, or myelofibrosis-free survival, $A S X L 1, S R S F 2$, and IDH2 variants/ mutations were identified as being "adverse" in PV and at least 1 of the 3 was present in 20 of the 133 patients (15\%). Patients with adverse variants/mutations displayed significantly worse survival when compared with both patients with other sequence variants/mutations $(H R$, $2.1 ; 95 \% \mathrm{Cl}, 1.1-4.1)$ and those without any sequence variant/mutation $(\mathrm{HR}, 2.2 ; 95 \% \mathrm{Cl}, 1.2-4.2$; Figure $2 \mathrm{~A})$; there was no difference in survival between patients with other sequence variants/mutations and those without any sequence variant $(P=.92)$.

The difference in survival between patients with and without adverse variants/mutations was independent of age, the IWG prognostic model for $\mathrm{PV},{ }^{17}$ and karyotype, with respective HRs (95\% Cls) of 2.7 (1.5-4.9), 2.8 (1.5-5.1), and 2.4 (1.2-4.6). Additional multivariable analysis that included the individual variables used in the IWG prognostic model ${ }^{17}$ confirmed the independent prognostic contribution of adverse variants/mutations ( $\mathrm{HR}, 3.0 ; 95 \% \mathrm{Cl}, 1.6-5.5)$, along with age $(\mathrm{HR}, 5.6 ; 95 \% \mathrm{Cl}, 3.1-10.0$ for age $\geq 65$ years $)$ and leukocytosis (HR, 2.0; 95\% Cl, 1.2-3.5 for leukocyte count $\geq 15 \times$ $10 \% / \mathrm{L})$; venous thrombosis was no longer significant in the particular multivariable model $(P=.32)$. The presence of adverse variants/mutations was also associated with shorter leukemiafree (Figure 3A; $P=.02 ; \mathrm{HR}, 5.6 ; 95 \% \mathrm{Cl}, 1.3-25.4$ ) and myelofibrosis-free (Figure $4 \mathrm{~A} ; P=.11 ; \mathrm{HR}, 2.5 ; 95 \% \mathrm{Cl}, 0.8-8.1$ ) survivals, although the latter did not reach significance.

Patients with 3 sequence variants/mutations $(n=4)$ had significantly worse survival, compared with those with $2(\mathrm{n}=26 ; P=.04)$, 1 ( $\mathrm{n}=40 ; P=.003)$, or no ( $\mathrm{n}=63 ; P=.003$ ) sequence variant/ mutation, whereas there was no difference in survival among the latter 3 groups $(P=.29)$, especially after adjusting for age $(P=.72)$. 


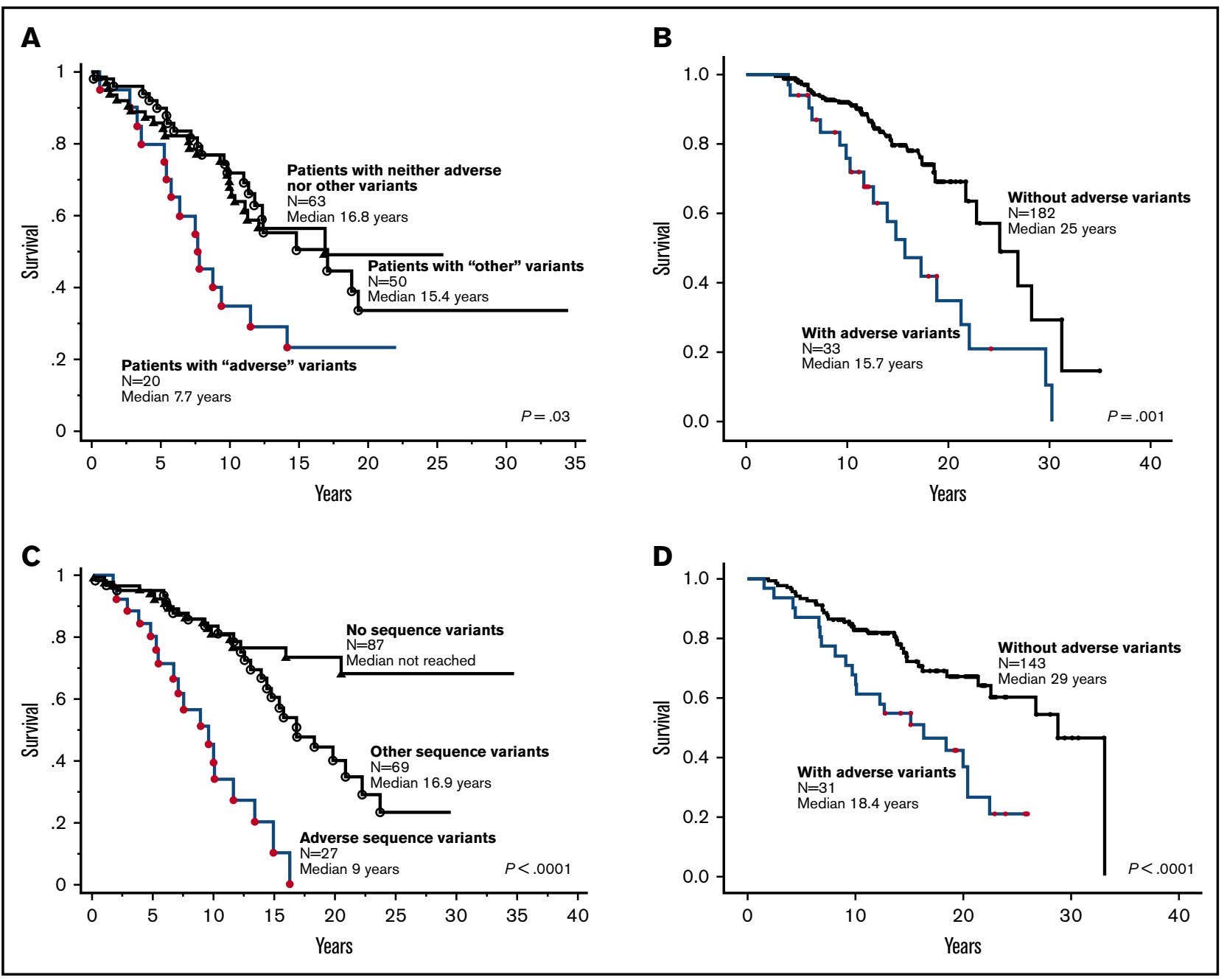

Figure 2. Overall survival curves. (A) Survival in 133 Mayo Clinic patients with PV stratified by the presence or absence of "adverse" (ASXL1, SRSF2, IDH2) or "other" (TET2, SH2B3, SF3B1, SETBP1, DNMT3A, CSF3R, CEBPA, SUZ12, ZRSR2, KIT, RUNX1, FLT3, CBL, and TP53) DNA sequence variants/mutations. (B) Survival in 215 Italian patients with PV stratified by the presence or absence of adverse (ASXL1, SRSF2, IDH2) variants/mutations. (C) Survival in 183 Mayo Clinic patients with ET stratified by the presence or absence of "adverse" (SH2B3, IDH2, SF3B1, U2AF1, EZH2, TP53) or "other" (TET2, ASXL1, PTP11, SUZ12, ZRSR2, CBL, CEBPA, CSF3R, DNMT3A, SRSF2, $F L T 3, K I T, N R A S, R U N X 1, S E T B P 1)$ sequence variants/mutations. (D) Survival in 174 Italian patients with ET stratified by the presence or absence of adverse (SH2B3, IDH2, $S F 3 B 1$, U2AF1, EZH2, TP53) variants/mutations.

Furthermore, the shorter survival seen in the 4 patients with 3 sequence variants/mutations was fully accounted for by the presence of adverse variants/mutations in all of them.

\section{Validation of prognostic relevance of adverse sequence variants/mutations in PV}

The observations from the Mayo Clinic patients were validated in an external cohort of 215 Italian patients with PV (Tables 1 and 2), followed for a median of 11.9 years and with 84 deaths (39\%), 55 fibrotic progressions (26\%), and 6 leukemic transformations (3\%); the particular cohort was enriched for patients who experienced fibrotic progression, in order to increase the power for molecular prediction for fibrotic transformation. In multivariable analysis, the unfavorable survival effect of adverse variants/mutations on survival (Figure 2B; HR, 2.6; 95\% Cl, 1.5-4.6) was independent of the IWG prognostic model for $\mathrm{PV}^{17}$; HRs (95\% Cls) were 2.3 (1.3-4.1) for adverse variants/mutations and 3.6 (1.7-7.8) for high- or intermediate-risk category. Adverse variants/mutations also affected myelofibrosis-free survival (Figure 4B), but the effect on leukemia-free survival was not significant (Figure $3 \mathrm{~B}$ ). Multivariable analysis confirmed the individual prognostic contribution of ASXL1 (HR, $2.41 ; 95 \% \mathrm{Cl}, 1.3-4.5)$ and SRSF2 ( $\mathrm{HR}, 3.84 ; 95 \% \mathrm{Cl}, 1.2-12.6)$ variants/mutations to overall survival and $A S X L 1$ to myelofibrosis-free survival (HR, 1.9; 95\% Cl, 1.2-3.6).

\section{Prognostic relevance of sequence variants/mutations in ET}

Median survival of the 183 Mayo Clinic patients with ET was 19.9 years. Age-adjusted univariate analysis identified $I D H 2, E Z H 2$, and $\mathrm{SH} 2 \mathrm{~B} 3$ variants/mutations as significant risk factors for survival; SH2B3 (HR, 3.0; 95\% Cl, 1.03-8.2) and IDH2 (HR, 22.1; 95\% Cl, 2.8-176.9) remained significant on multivariable analysis. For leukemia-free and myelofibrosis-free survival, univariate analysis showed significant associations with TP53, EZH2, SRSF2, and 


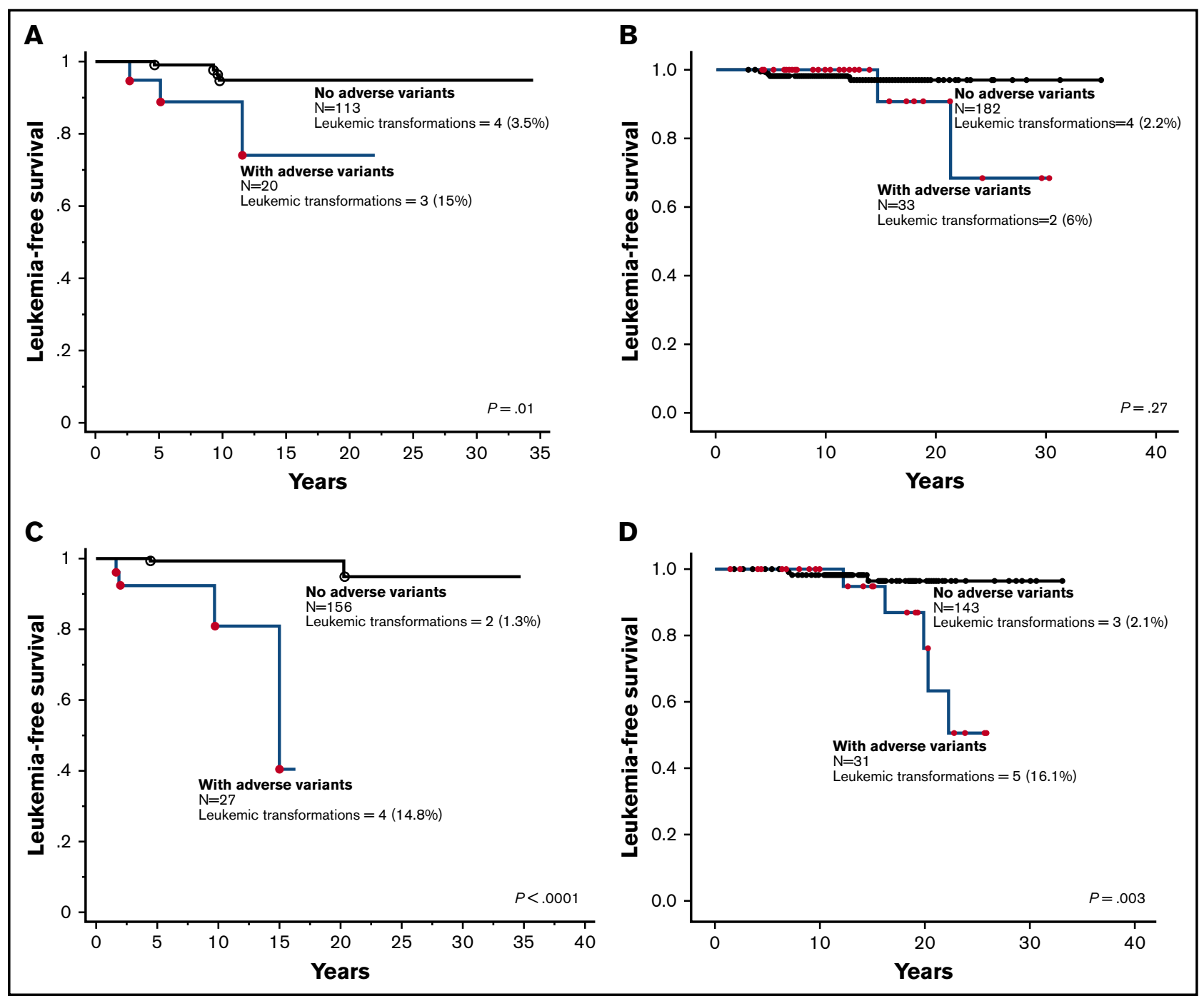

Figure 3. Leukemia-free survival curves. (A) Leukemia-free survival in 133 Mayo Clinic patients with PV stratified by the presence or absence of "adverse" (ASXL1, $S R S F 2, I D H 2)$ DNA sequence variants/mutations. (B) Leukemia-free survival in 215 Italian patients with PV stratified by the presence or absence of adverse variants/mutations. (C) Leukemia-free survival in 183 Mayo Clinic patients with ET stratified by the presence or absence of "adverse" (SH2B3, IDH2, SF3B1, U2AF1, EZH2, TP53) sequence variants/mutations. (D) Leukemia-free survival in 174 Italian patients with ET stratified by the presence or absence of adverse sequence variants/mutations.

IDH2 variants/mutations for the former and with $S F 3 B 1$ and U2AF1 for the latter; on multivariable analysis, TP53 (HR, 82.8; 95\% Cl, 7.5-916) and EZH2 (HR, 146.8; 95\% Cl, 11.1-1935.6) remained significant for leukemia-free survival and SF3B1 (HR, 8.1; 95\% Cl, 2.5-25.8) and U2AF1 (HR, 30.3; 95\% Cl, 3.4-271.0) for myelofibrosis-free survival.

Based on their above-outlined independent impact on overall, leukemia-free, or myelofibrosis-free survival, $S H 2 B 3, I D H 2, U 2 A F 1$, SF3B1, EZH2, and TP53 variants/mutations were identified as being "adverse" and at least 1 of them was present in 27 (14.8\%) of the 183 patients with ET. Patients with adverse variants/mutations displayed significantly worse overall survival, when compared with both patients with other sequence variants/mutations (HR, 4.3; $95 \% \mathrm{Cl}, 2.2-8.5)$ and those without any sequence variant/mutation (HR, 7.0; 95\% Cl, 3.5-14.1; Figure 2C). In univariate analysis, patients with other sequence variants/mutations also showed significantly worse survival, when compared with patients without any sequence variant/mutation ( $\mathrm{HR}, 2.0 ; 95 \% \mathrm{Cl}, 1.1-3.7)$; however, in multivariable analysis that included age, the difference in survival remained significant for patients with adverse variants/mutations (HR, $3.4 ; 95 \% \mathrm{Cl}, 1.6-7.0)$ but not for those with other sequence variants/ mutations ( $\mathrm{HR}, 1.5 ; 95 \% \mathrm{Cl}, 0.8-2.8)$. The presence of adverse variants/mutations was also associated with significantly shorter leukemia-free (Figure 3C; HR, 36.2; 95\% Cl, 3.8-347.9) and myelofibrosis-free (Figure 4C; HR, 6.6; 95\% Cl, 2.6-16.4) survivals.

Patients without any sequence variant/mutation $(n=87)$ had significantly longer survival when compared with those with 3 or 4 ( $\mathrm{n}=7 ; P<.0001)$ or $1(\mathrm{n}=75 ; P=.0009)$ sequence variant but not to those with 2 sequence variants ( $\mathrm{n}=14 ; P=.28$ ). However, when analysis was repeated after adjusting for age and excluding patients with adverse variants/mutations $(n=27)$, the survival impact of the number of mutations was no longer evident $(P=.21)$; 6 of the 7 patients with 3 or more sequence variants also had adverse variants. In other words, in both ET and PV, patients with 


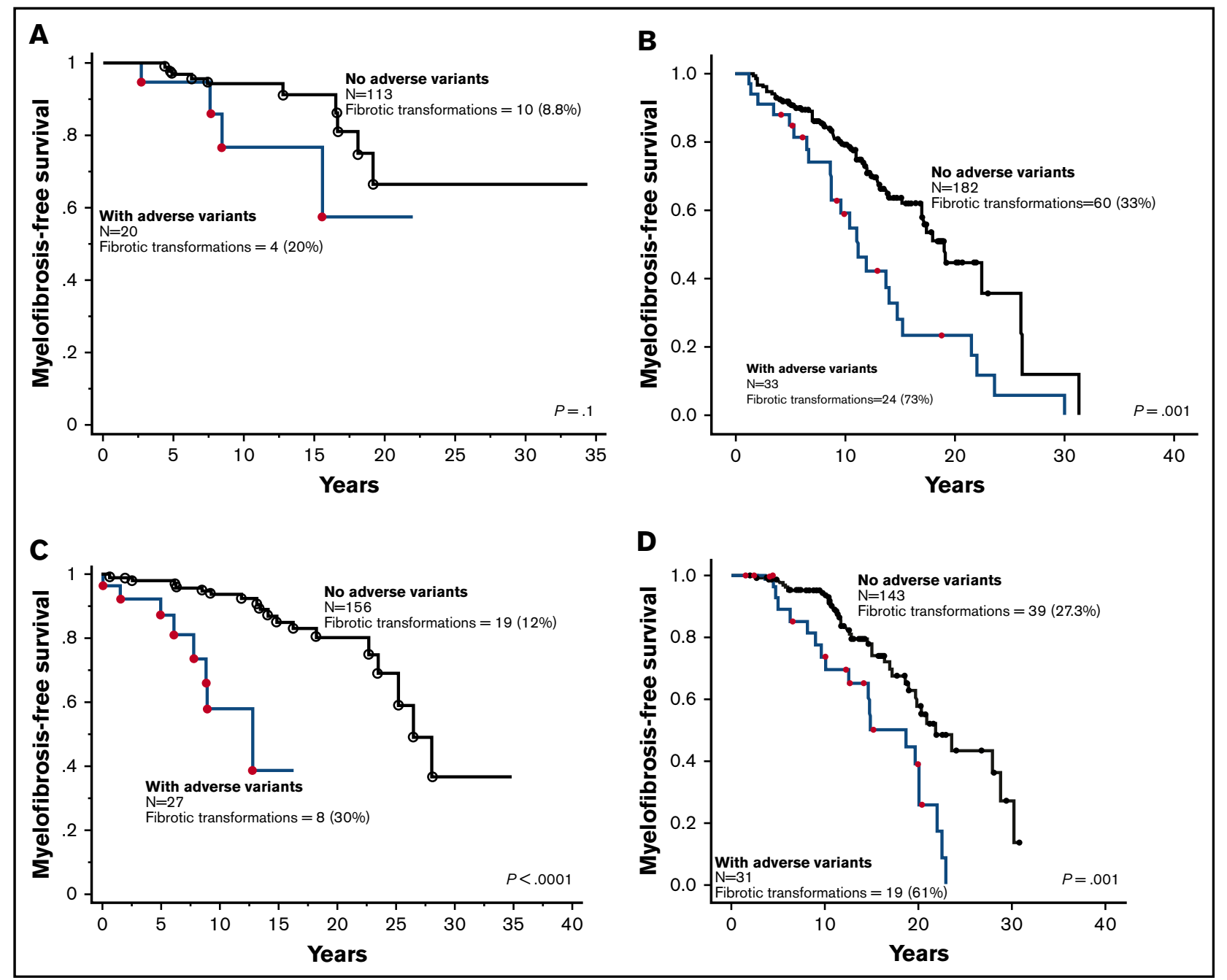

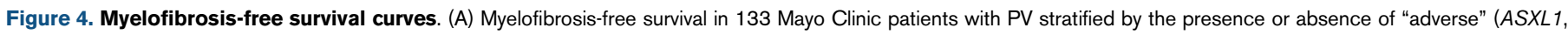

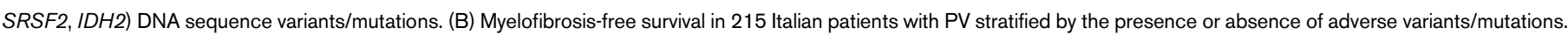

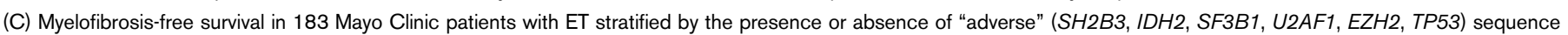
variants/mutations. (D) Myelofibrosis-free survival in 174 Italian patients with ET stratified by the presence or absence of adverse sequence variants/mutations.

multiple sequence variants were older and more likely to harbor adverse variants, thus giving the appearance of unfavorable prognostic impact during unadjusted survival analysis.

The difference in survival between patients with and without adverse variants/mutations was independent of age ( $\mathrm{HR}, 2.6 ; 95 \% \mathrm{Cl}, 1.4-4.8)$, IWG prognostic model for $\mathrm{ET}^{16}(\mathrm{HR}, 2.6 ; 95 \% \mathrm{Cl}, 1.4-4.8)$, and karyotype (HR, 4.7; 95\% Cl, 2.4-9.2). Additional multivariable analysis that included the individual variables used in the IWG prognostic model ${ }^{16}$ confirmed the independent prognostic contribution of adverse variants/mutations $(\mathrm{HR}, 2.4 ; 95 \% \mathrm{Cl}, 1.2-4.7)$, along with age $(\mathrm{HR}, 3.2$; $95 \% \mathrm{Cl}, 1.6-6.2$ for age $\geq 60$ years) and leukocytosis (HR, 2.1; 95\% $\mathrm{Cl}, 1.2-3.7$ for leukocyte count $\left.\geq 11 \times 10^{9} / \mathrm{L}\right)$; thrombosis was no longer significant in the particular multivariable model $(P=.68)$.

\section{Validation of prognostic relevance of adverse sequence variants/mutations in ET}

The observations from the Mayo Clinic patients were validated in an external cohort of 174 Italian patients with ET (Tables 1 and 2) followed for a median of 13 years and with 62 deaths (36\%), 58 fibrotic progressions (33\%), and 8 leukemic transformations (5\%); the particular cohort was enriched for patients who experienced fibrotic progression, in order to increase the power for molecular prediction for myelofibrosisfree survival. In multivariable analysis, the unfavorable survival effect of adverse variants/mutations (Figure 2D; HR, 2.5; 95\% Cl, 1.5-4.4) was independent of the IWG prognostic model for $\mathrm{ET}^{16}$ : HRs $(95 \% \mathrm{Cls}$ ) were 2.0 (1.1-3.6) for adverse variants, 3.5 (1.6-7.7) for the intermediaterisk category, and 8.7 (3.6-21.0) for the high-risk category. Multivariable analysis confirmed the individual prognostic contribution of U2AF1 to overall (HR, 2.9; 95\% Cl, 1.1-8.7) and myelofibrosis-free (HR, 3.2; 95\% $\mathrm{Cl}, 1.1-9.0)$ survival and that of TP53 to leukemia-free survival (HR, 7.3; $95 \% \mathrm{Cl}, 1.5-35.9)$. Adverse variants/mutations also affected leukemiafree (Figure 3D; HR, 6.7; 95\% Cl, 1.6-28.1) and myelofibrosis-free survival (Figure 4D; HR, 2.6; 95\% Cl, 1.4-4.5).

\section{Discussion}

The revelation, in the current study, of DNA sequence variants/ mutations, other than JAK2/CALR/MPL, in the majority of patients 
with PV or ET is consistent with the results from a smaller previous study that showed the presence of proven mutations in $44 \%$ and $29 \%$ of the respective diseases. ${ }^{8}$ The current study identifies prognostically important specific sequence variants/mutations and demonstrates the subsidiary role, in this regard, of the "number" of mutations.

The detrimental nature of some of the adverse variants identified in the current study has also been recognized in other chronic myeloid malignancies, including PMF (ASXL1, SRSF2, EZH2, IDH1/2, U2AF1), ${ }^{7,18}$ chronic myelomonocytic leukemia $(A S X L 1),{ }^{19}$ and myelodysplastic syndromes ( $A S X L 1, E Z H 2, T P 53, S R S F 2) .{ }^{20,21}$ Similarly, TP53, IDH2, SRSF2, and $S H 2 B 3$ mutations were previously found to be overrepresented in blast-phase MPNs. ${ }^{8,22,23}$ In this regard, the possible leukemogenic cooperation of mutant JAK2 with IDH1/2 mutations has previously been clinically suggested $^{24}$ and that of TP53 loss with mutant JAK2 demonstrated in vivo. ${ }^{23}$ Consistent with the latter observation, all 5 TP53-mutated patients with $\mathrm{ET}$, in the current study, were also JAK2-mutated, and leukemic transformation in another study ${ }^{8}$ was temporally linked to the consequent loss of the wild-type TP53 allele in TP53-mutated patients.

The observations from the current study are practically relevant and timely considering the increasing use of targeted NGS in routine myeloid cancer practice. We show that the occurrence of sequence variants/mutations other than JAK2/CALR/MPL, in patients with $\mathrm{PV}$ or $\mathrm{ET}$, is not infrequent and neither the type nor number of genes involved is necessarily detrimental to disease outcome, unless it involves specific genes with prognostic relevance. On the other hand, patients with adverse variants/mutations, regardless of their conventional risk category, might require closer monitoring. In this regard, the current study suggests the possibility of refining clinically derived prognostic models by the incorporation of molecular information.

Additional studies are warranted in order to refine and possibly expand the pool of clinically relevant DNA sequence variants/ mutations that could be targeted by NGS. Such a strategy would ideally also include sequence variants/mutations that identify patients with increased risk of thrombosis. In this regard, the discrepant results between the Mayo Clinic and Florence patient cohorts, in regards to the possible association between $T E T$ variants/mutations and thrombosis in ET, remains unexplained and deserves further investigation.

\section{Acknowledgments}

This work was supported in part by The Mayo Clinic Harvey-Yulman Charitable Foundation for Myelofibrosis Tissue Bank and Clinical Database of Molecular and Biological Abnormalities, The Henry J. Predolin Foundation for Research in Leukemia, Mayo Clinic, Rochester, MN. The work in Florence was supported by Associazione Italiana per la Ricerca sul Cancro (AIRC) "Special Program Molecular Clinical Oncology $5 \times 1000$ " to the AIRC-Gruppo Italiano Malattie Mieloproliferative (AGIMM) group (project \#1005; a detailed description of the AGIMM project is available at http://www.progettoagimm.it). P.G. was supported by GR-2011-02352109 Bando Ministero della Salute Giovani Ricercatori-Ricerca Finalizzata 2011-2012 and by AIRC IG-15967. G.R. was the recipient of a fellowship from Amici di Beat Leukemia Onlus.

\section{Authorship}

Contribution: A.T. designed and sponsored the study, contributed patients, collected clinical data and patient samples, reviewed the molecular data, performed statistical analysis, and wrote the paper; T.L.L. performed and analyzed the molecular analysis; P.G. contributed patients, collected clinical data and patient samples, performed and analyzed the molecular data, performed statistical analysis, and helped with manuscript writing; C.M.F. performed the molecular analysis; G.R., A. Pacilli, A. Pancrazzi, C.M., and T.F. performed sequencing and analysis of data; Y.E. and D.B. collected clinical data; C.A.H. performed pathology review; R.P.K. performed cytogenetics review; A. Pardanani contributed patients and reviewed the molecular data; N.G. contributed patients and collected clinical data; A.M.V. designed the study, contributed patients, collected clinical data and patient samples, reviewed the molecular data, and helped with data analysis and manuscript writing; and all authors reviewed and approved the paper.

Conflict-of-interest disclosure: The authors declare no competing financial interests.

Correspondence: Ayalew Tefferi, Department of Internal Medicine, Division of Hematology, Mayo Clinic College of Medicine, 200 First St SW, Rochester, MN 55905; e-mail: tefferi.ayalew@mayo.edu.

\section{References}

1. James C, Ugo V, Le Couédic JP, et al. A unique clonal JAK2 mutation leading to constitutive signalling causes polycythaemia vera. Nature. 2005; 434(7037):1144-1148.

2. Scott LM, Tong W, Levine RL, et al. JAK2 exon 12 mutations in polycythemia vera and idiopathic erythrocytosis. N Engl J Med. 2007;356(5):459-468.

3. Pikman Y, Lee BH, Mercher T, et al. MPLW515L is a novel somatic activating mutation in myelofibrosis with myeloid metaplasia. PLoS Med. 2006;3(7):e270.

4. Delhommeau F, Dupont S, Della Valle V, et al. Mutation in TET2 in myeloid cancers. N Engl J Med. 2009;360(22):2289-2301.

5. Klampfl T, Gisslinger H, Harutyunyan AS, et al. Somatic mutations of calreticulin in myeloproliferative neoplasms. N Engl J Med. 2013;369(25): 2379-2390.

6. Nangalia J, Massie CE, Baxter EJ, et al. Somatic CALR mutations in myeloproliferative neoplasms with nonmutated JAK2. N Engl J Med. 2013;369(25): 2391-2405.

7. Vannucchi AM, Lasho TL, Guglielmelli P, et al. Mutations and prognosis in primary myelofibrosis. Leukemia. 2013;27(9):1861-1869.

8. Lundberg P, Karow A, Nienhold R, et al. Clonal evolution and clinical correlates of somatic mutations in myeloproliferative neoplasms. Blood. 2014; 123(14):2220-2228.

9. Tefferi A, Pardanani A. Myeloproliferative Neoplasms: A Contemporary Review. JAMA Oncol. 2015;1(1):97-105. 
10. Barbui T, Thiele J, Vannucchi AM, Tefferi A. Rationale for revision and proposed changes of the WHO diagnostic criteria for polycythemia vera, essential thrombocythemia and primary myelofibrosis. Blood Cancer J. 2015;5:e337.

11. Tefferi A, Lasho TL, Finke CM, et al. CALR vs JAK2 vs MPL-mutated or triple-negative myelofibrosis: clinical, cytogenetic and molecular comparisons. Leukemia. 2014;28(7):1472-1477.

12. Tefferi A, Lasho TL, Tischer A, et al. The prognostic advantage of calreticulin mutations in myelofibrosis might be confined to type 1 or type 1 -like CALR variants. Blood. 2014;124(15):2465-2466.

13. Barbui T, Finazzi G, Carobbio A, et al. Development and validation of an international prognostic score of thrombosis in World Health Organizationessential thrombocythemia (IPSET-thrombosis). Blood. 2012;120(26):5128-5133.

14. Guglielmelli $P$, Lasho TL, Rotunno G, et al. The number of prognostically detrimental mutations and prognosis in primary myelofibrosis: an international study of 797 patients. Leukemia. 2014;28(9):1804-1810.

15. Vardiman JW, Thiele J, Arber DA, et al. The 2008 revision of the World Health Organization (WHO) classification of myeloid neoplasms and acute leukemia: rationale and important changes. Blood. 2009;114(5):937-951.

16. Passamonti F, Thiele J, Girodon F, et al. A prognostic model to predict survival in 867 World Health Organization-defined essential thrombocythemia at diagnosis: a study by the International Working Group on Myelofibrosis Research and Treatment. Blood. 2012;120(6):1197-1201.

17. Tefferi A, Rumi E, Finazzi G, et al. Survival and prognosis among 1545 patients with contemporary polycythemia vera: an international study. Leukemia. 2013;27(9):1874-1881.

18. Tefferi A, Finke CM, Lasho TL, et al. U2AF1 mutations in primary myelofibrosis are strongly associated with anemia and thrombocytopenia despite clustering with JAK2V617F and normal karyotype. Leukemia. 2014;28(2):431-433.

19. Patnaik MM, Itzykson R, Lasho TL, et al. ASXL1 and SETBP1 mutations and their prognostic contribution in chronic myelomonocytic leukemia: a two-center study of 466 patients. Leukemia. 2014;28(11):2206-2212.

20. Bejar R, Stevenson K, Abdel-Wahab O, et al. Clinical effect of point mutations in myelodysplastic syndromes. N Engl J Med. 2011;364(26):2496-2506.

21. Thol F, Kade S, Schlarmann C, et al. Frequency and prognostic impact of mutations in SRSF2, U2AF1, and ZRSR2 in patients with myelodysplastic syndromes. Blood. 2012;119(15):3578-3584.

22. Pardanani A, Lasho T, Finke C, Oh ST, Gotlib J, Tefferi A. LNK mutation studies in blast-phase myeloproliferative neoplasms, and in chronic-phase disease with TET2, IDH, JAK2 or MPL mutations. Leukemia. 2010;24(10):1713-1718.

23. Rampal R, Ahn J, Abdel-Wahab O, et al. Genomic and functional analysis of leukemic transformation of myeloproliferative neoplasms. Proc Natl Acad Sci USA. 2014;111(50):E5401-E5410.

24. Tefferi A, Jimma T, Sulai $\mathrm{NH}$, et al. IDH mutations in primary myelofibrosis predict leukemic transformation and shortened survival: clinical evidence for leukemogenic collaboration with JAK2V617F. Leukemia. 2012;26(3):475-480. 